\title{
Measurement and Analysis of Neutron Leakage Spectra from Pb and LBE Cylinders with D-T Neutrons
}

\author{
Size Chen ${ }^{1}$, Leting Gan ${ }^{1,2}$, Taosheng $\mathrm{Li}^{1}$, Yuncheng Han ${ }^{1}$, Chao Liu',a , Jieqiong Jiang ${ }^{1}$ and Yican $\mathrm{Wu}^{1,2}$
}

${ }^{1}$ Key Laboratory of Neutronics and Radiation Safety, Institute of Nuclear Energy Safety Technology, Chinese Academy of Sciences, Hefei, Anhui, 230031, China

${ }^{2}$ University of Science and Technology of China, Hefei, Anhui, 230027, China

\begin{abstract}
For validating the current evaluated neutron data libraries, neutron leakage spectra from lead and lead bismuth eutectic (LBE) cylinders have been measured using an intense D-T pulsed neutron source with time-of-flight (TOF) method by Institute of Nuclear Energy Safety Technology (INEST), Chinese Academy of Sciences (CAS). The measured leakage spectra have been compared with the calculated ones using Super Monte Carlo Simulation Program for Nuclear and Radiation Process (SuperMC) with the evaluated pointwise data of lead and bismuth processed from ENDF/B-VII.1, JEFF-3.1 and JENDL-4.0 libraries. This work shows that calculations of the three libraries are all generally consistent with the lead experimental result. For LBE experiment, the JEFF-3.1 and JENDL-4.0 calculations both agree well with the measurement. However, the result of ENDF/B-VII.1 fails to fit with the measured data, especially in the energy range of 5.5 and $7 \mathrm{MeV}$ with difference more than $80 \%$. Through sensitivity analysis with partial cross sections of ${ }^{209} \mathrm{Bi}$ in ENDF/B-VII.1 and JEFF, the difference between the measurement and the ENDF/B-VII.1 calculation in LBE experiment is found due to the neutron data of ${ }^{209} \mathrm{Bi}$.
\end{abstract}

\section{INTRODUCTION}

Lead bismuth eutectic (LBE) alloy plays an important role in many advanced nuclear applications such as accelerator driven subcritical system (ADS)[1-3]. It is necessary to perform a new integral experiment on $\mathrm{Pb}$ and $\mathrm{Bi}$ to validate the current evaluated neutron data libraries for the design of ADS.

\section{EXPERIMENT PROCEDURE}

This experiment was performed at a Cockcroft-Waltontype neutron generator installed at China Institute of Atomic Energy (CIAE) [4]. It can be used for both continuous and pulsed beam operations to generate neutrons for various kinds of experiments. In this work, the pulsed deuteron beam was accelerated up to $300 \mathrm{keV}$ and bombarded an air-cooling tritium target to yield $14.8 \mathrm{MeV}$ neutrons with 3 ns FWHM (full width at half maximum). The repetition rate was $1.5 \mathrm{MHz}$ and the average deuteron current was $30 \mu \mathrm{A}$, which produced a neutron intensity of about $10^{9} \mathrm{n} / \mathrm{s}$.

A stilbene crystal detector was positioned at $7.05 \pm 0.01$ $\mathrm{m}$ from the neutron source at forward direction to check the accelerator pulsed mode operation. The leakage neutron spectra were measured using a BC501A $(\varnothing 5.08 \times$ $2.54 \mathrm{~cm}$ ) liquid scintillation detector by time of flight
(TOF) technique.

The process of the leakage neutron spectra measuring included the sample in and sample out measurements. The sample in measurement and sample out survey obtained the foreground and background data, respectively. The net TOF spectra were achieved by subtracting the background data from the foreground data. The uncertainties of the experimental data contain the statistical uncertainty $(<5 \%)$, the neutron detection efficiency $(\sim 3 \%)$ and the neutron source yield $(\sim 2 \%)$.

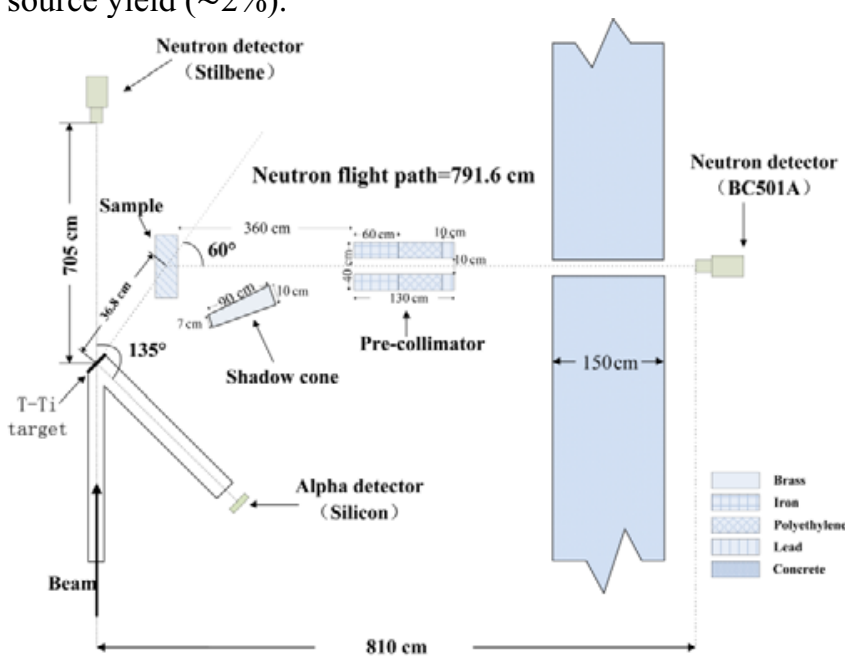

Figure 1. Experimental Arrangement

a Corresponding author: chao.liu@fds.org.cn 


\section{METHOD OF CALCULATION}

The calculated leakage spectra were gained by using SuperMC[5] with lead and bismuth pointwise data from the ENDF/B-VII.1[6], JEFF-3.1[7] and JENDL-4.0 [8] libraries.

In this simulation, source neutron was assumed to be a point source. And a point detector was used at the position of $791.6 \mathrm{~cm}$ from the sample $(\mathrm{Pb} / \mathrm{LBE})$. The angle dependent neutron energy spectra of the neutron source, the energy efficiency of the BC501A detector and the width of the beam pulse were considered in order to accord with the actual situation of the experiment. The total neutron histories in the model were 109, and the average error is about $0.5 \%$.

\section{RESULTS AND DISCUSSION}

Figures 2-3 show the comparisons between the measured and simulated spectra on $\mathrm{Pb}$ and LBE samples, respectively.

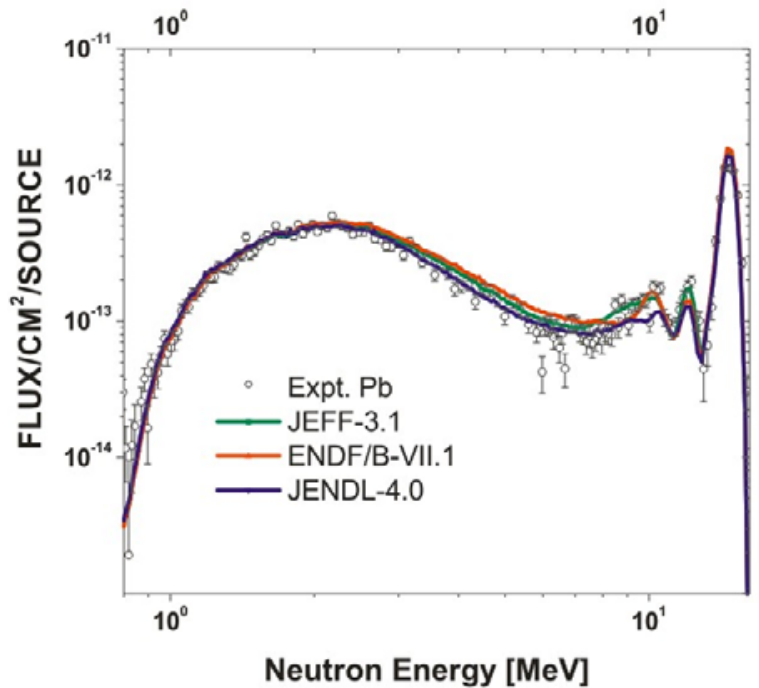

Figure 2. Measured and calculated neutron spectra of $\mathrm{Pb}$

Each spectrum was normalized to the source neutron. To search for differences between calculations and measurements in detail, each energy spectrum was divided into groups by integral of the flux in the following energy regions; (A) $16>\mathrm{E}_{\mathrm{n}}>10.5 \mathrm{MeV}$, (B) $10.5>\mathrm{E}_{\mathrm{n}}>7 \mathrm{MeV}$, (C) $7>\mathrm{E}_{\mathrm{n}}>5.5 \mathrm{MeV}$, (D) $5.5>\mathrm{E}_{\mathrm{n}}>2.5 \mathrm{MeV}$, (E) $2.5>\mathrm{En}>1.2$ $\mathrm{MeV}$, (F) $1.2>\mathrm{En}>0.8 \mathrm{MeV}$. Calculation-to-experiment ratios $(\mathrm{C} / \mathrm{E})$ of the $\mathrm{Pb}$ and $\mathrm{LBE}$ spectra are listed in Table 1 and Table 2, respectively.

Table 1: $\mathrm{C} / \mathrm{E}$ values for $\mathrm{Pb}$ in different energy intervals

\begin{tabular}{cccc}
\hline \multirow{2}{*}{$\begin{array}{c}\text { Energy region } \\
(\mathrm{MeV})\end{array}$} & \multicolumn{3}{c}{$\mathbf{C} / \mathbf{E}$} \\
\cline { 2 - 4 } & JEFF-3.1 & ENDF/B-VII.1 & JENDL-4.0 \\
\hline $16>\mathrm{En}>10.5$ & 1.039 & 1.080 & 0.963 \\
$10.5>\mathrm{En}>7$ & 1.092 & 1.044 & 0.871 \\
$7>\mathrm{En}>5.5$ & 1.124 & 1.158 & 0.944 \\
$5.5>\mathrm{En}>2.5$ & 1.113 & 1.188 & 1.013 \\
$2.5>\mathrm{En}>1.2$ & 0.995 & 1.027 & 1.003 \\
$1.2>\mathrm{En}>0.8$ & 0.963 & 0.943 & 1.020 \\
\hline
\end{tabular}

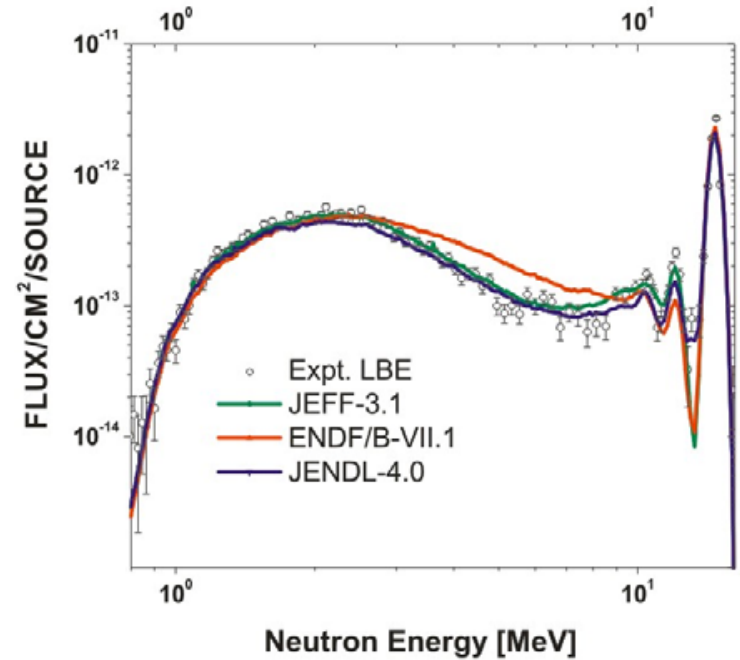

Figure 3: Measured and calculated neutron spectra of LBE

As shown in Figure 2 and Table 1 for $\mathrm{Pb}$ sample, in the energy range of 10.5-16 MeV, discrepancies between calculations of three libraries and experiment exceed no more than $8 \%$. In 7-10.5 MeV region, the result of JENDL4.0 underestimates nearly $13 \%$, while the $\mathrm{C} / \mathrm{E}$ values of JEFF-3.1 and ENDF/B-VII.1 are 1.092 and 1.044 respectively. The difference between the partial spectrum calculated with JENDL-4.0 and obtained from experiment doesn't exceed $6 \%$ in the energy range between 2.5 and 7 $\mathrm{MeV}$, and calculated results from JEFF-3.1 and ENDF/BVII. 1 in the same energy range overestimate that from measurement $10 \%$ and $15 \%$, respectively. When neutron energy is less than $2.5 \mathrm{MeV}$, all of three calculations exceed the measurement less than $6 \%$. In general, calculated results obtained from ENDF/B-VII.1, JEFF-3.1 and JENDL-4.0 agree well with experimental result and the conclusion consists with Chikara Konno's evaluation on JENDL-4.0, while small discrepancies existed in partial energy interval.

Table 2: $\mathrm{C} / \mathrm{E}$ values for $\mathrm{LBE}$ in different energy intervals

\begin{tabular}{cccc}
\hline \multirow{2}{*}{$\begin{array}{c}\text { Energy region } \\
(\mathbf{M e V})\end{array}$} & \multicolumn{3}{c}{ C/E } \\
\cline { 2 - 4 } & JEFF-3.1 & ENDF/B-VII.1 & JENDL-4.0 \\
\hline $16>$ En $>10.5$ & 1.012 & 1.050 & 1.011 \\
$10.5>\operatorname{En}>7$ & 1.120 & 1.195 & 0.913 \\
$7>\mathrm{En}>5.5$ & 1.144 & 1.803 & 1.051 \\
$5.5>\mathrm{En}>2.5$ & 1.020 & 1.265 & 0.899 \\
$2.5>\mathrm{En}>1.2$ & 0.968 & 0.893 & 0.880 \\
$1.2>\mathrm{En}>0.8$ & 1.026 & 0.892 & 1.021 \\
\hline
\end{tabular}

Figure 3 and Table 2 give the result of $\mathrm{LBE}$ measurement. It is found that the simulation of JEFF-3.1 overestimates the experimental result $12-15 \%$ in the energy region of 5.5-10.5 MeV. In other energy range, the differences are within $4 \%$. The result with JENDL-4.0 library underestimates the measurement by $10-12 \%$ in the 1.2-5.5 MeV range. For other energy regions, the difference between the calculation and the experiment is less than $10 \%$. However, the discrepancy between ENDF/B-VII.1 calculation and the experimental spectrum is more than $10 \%$ in the energy group, $0.8-10.5 \mathrm{MeV}$, and 
nearly $80 \%$ in the interval $5.5-7 \mathrm{MeV}$. In $10.5-16 \mathrm{MeV}$ energy range, the $\mathrm{C} / \mathrm{E}$ value of ENDF/B-VII.1 is 1.050 . From the above observation, it can be seen that the simulations of JEFF-3.1 and JENDL-4.0 agree with the experimental data much better than calculation of ENDF/B-VII.1 in general. Drastic overestimation is observed in the ENDF/B-VII.1 calculation by a factor of 1.8 as compared to the experimental result in the 5.5-7 $\mathrm{MeV}$ range.

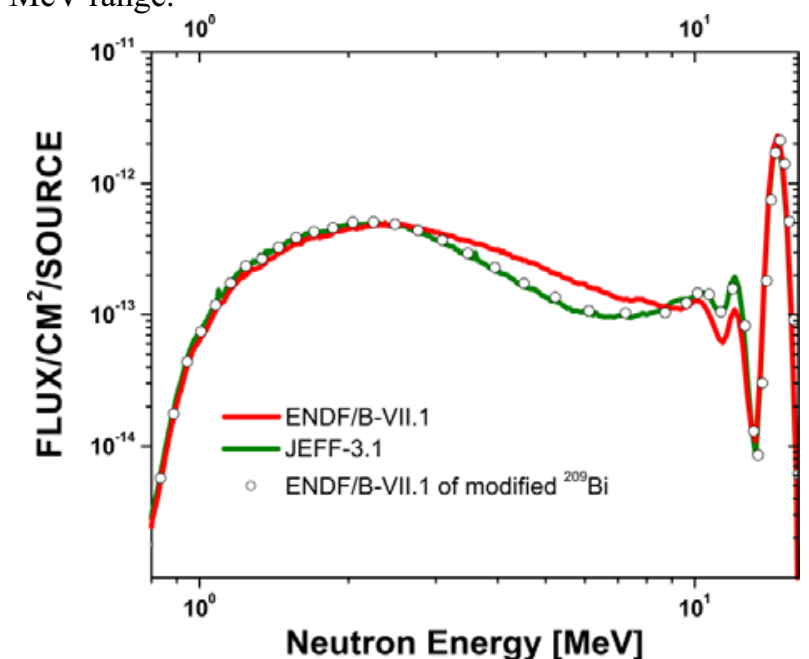

Figure 4: Calculation spectra dependence of modified ${ }^{209} \mathrm{Bi}$ cross section from ENDF/B-VII.1 to JEFF-3.1

To clarify the big difference between ENDF/B-VII.1 and measurement in the 2.5-7 MeV energy region on LBE experiment, more calculations using JEFF-3.1 and ENDF/B-VII.1 were made. Calculation was taken with modified ENDF/B-VII.1 library which replaced the data of ${ }^{209} \mathrm{Bi}$, the only nature nuclide of bismuth, from ENDF/BVII.1 to JEFF-3.1. The result is similar to the JEFF-3.1 calculation as shown in Figure 4. From the above comparison, we find that the difference between the measurement and the ENDF/B-VII.1 calculation in LBE experiment is really due to the neutron data of ${ }^{209} \mathrm{Bi}$ as the calculation with JEFF-3.1 agrees well with the experimental result.

\section{ACKNOWLEDGMENT}

This work was supported by the Natural Science Foundation of China, Grant No. 91026004. We further thank the great help from members of FDS Team and from researchers and engineers of CIAE in this work.

\section{References}

1. C. Bowman, E. Arthur, P. Lisowski, et al., Nucl. Instrum. and Meth. A, 320.1, 336-367 (1992)

2. S. Kapoor, Pramana, 59.6, 941-950 (2002)

3. P. Gokhale, S. Deokattey, V. Kumar, Prog. in Nucl. Energy, 48.2, 91-102 (2006)

4. J.Q. Lu, D.L. Xie, S.W. Quan, et al., Atom. Energy Sci. and Tech., 37.4, 376-380 (2003)

5. Y.C. Wu, J. Song, H. Zheng, et al., Ann Nucl. Energy, 82, 161-168 (2015)
6. M. Chadwick, M. Herman, P. Obložinský, et al., Nucl. Data Sheets, 112.12, 2887-2996 (2011)

7. A. Santamarina, D. Bernard, P. Blaise, et al., JEFF Report, 22.10.2, 2 (2009)

8. K. Shibata, O. Iwamoto, T. Nakagawa, et al., J. Nucl. Sci. and Tech., 48.1, 1-30 (2011) 